\title{
RESISTÊNCIA DE PARASITOS GASTRINTESTINAIS DE OVINOS A ALGUNS ANTI-HELMÍNTICOS NO ESTADO DE SANTA CATARINA, BRASIL
}

\author{
GASTRO-INTESTINAL PARASITES RESISTANCE IN SHEEP TO SOME \\ ANTHELMINTICS IN SANTA CATARINA STATE, BRAZIL
}

\author{
César Itaqui Ramos ${ }^{1}$ Valdomiro Bellato ${ }^{2}$ Volney Silveira de Ávila ${ }^{1}$ \\ Guilherme Caldeira Coutinho ${ }^{1}$ Antonio Pereira de Souza ${ }^{2}$
}

RESUMO

A atuação dos anti-helmínticos à base de ivermectin $(0,2 \mathrm{mg} / \mathrm{kg})$, levamisole (10mg/kg), closantel (10mg/kg) e albendazole (10mg/kg), foi avaliada em 65 rebanhos ovinos do estado de Santa Catarina, Brasil. As avaliações foram executadas quando o rebanho apresentava-se com o lote controle acima de 500 ovos por grama de fezes (OPG) da ordem Strongylida, acompanhado do cultivo de larvas. Foram aleatoriamente formados quatro grupos de 10 animais, identificados e tratados com os respectivos anti-helmínticos. Sete a 10 dias após, foram coletadas amostras de fezes dos animais de cada grupo para a verificação do OPG e identificação das larvas e os resultados comparados com o do lote controle. Considerou-se resistência quando a eficácia da droga foi menor do que $95 \%$ e o intervalo de confiança, menor do que 90\%. Dos 65 rebanhos avaliados, a resistência ao ivermectin esteve presente em $77 \%$, sendo somente identificadas larvas de Haemonchus (100\%), ao albendazole em $65 \%$, sendo Haemonchus (74\%), Ostertagia (15\%) e Trichostrongylus (11\%), ao closantel em 13\%, sendo Haemonchus (100\%) e em $15 \%$ ao levamisole sendo Trichostrongylus (44\%), Ostertagia (39\%) e Haemonchus (17\%). Conclui-se que a multi-resistência está presente na maioria do rebanho ovino catarinense.

Palavras-chave: anti-helmínticos, nematóides, resistência ovinos.

\section{SUMMARY}

Sixty-five sheep flocks were studied in Santa Catarina State to determine parasites resistance to anthelmintics based on ivermectin $(0.2 \mathrm{mg} / \mathrm{kg})$, levamisole $(10 \mathrm{mg} / \mathrm{kg})$, closantel $(10 \mathrm{mg} / \mathrm{kg})$ and albendazole $(10 \mathrm{mg} / \mathrm{kg})$. The evaluation was carryed out when a particular sheep flock showed a control group with EPG (eggs per gram) level above 500. Larvae culture was also performed to allow the parasite identification. Four

\begin{abstract}
randomized groups of 10 animals were formed and treated with one of the four anthelmintic products. Faecal samples were collected of each group seven to ten days after the treatment, aiming to perform the eggs count and larvae identification. These results were compared with results of the control group. The anthelmintics were considered to present evidence of parasite resistance when the efficacy of the product was lower than $95 \%$. From the total of 65 sheep flock studied, seventy-seven percent presented parasite resistance to ivermectin, with Haemonchus larvae only (100\%); sixty-five percent to albendazole, with Haemonchus (74\%), Ostertagia (15\%) and Trichostrongylus (11\%); thirteen percent to closantel, with Haemonchus (100\%); and fifteen percent to levamisole, with Thichostrongylus (44\%), Ostertagia (39\%) and Haemonchus (17\%). The results detected the presence of a multi-resistance to anthelminthics in the great majority of the sheep flocks of Santa Catarina State.
\end{abstract}

Key words: anthelmintics, nematodes, resistance, sheep.

\section{INTRODUÇÃO}

A ovinocultura, em Santa Catarina, está presente em grande parte do Estado, principalmente em pequenas propriedades, com rebanhos na grande maioria abaixo de 50 animais, totalizando 250.000 ovinos (IBGE, 1997). No aspecto de saúde, as helmintoses gastrintestinais são os principais problemas. O uso intensivo de anti-helmínticos, muitas vezes, em subdoses, aliado a problemas de manejo, tem selecionado estirpes resistentes a vários produtos, principalmente Haemonchus spp., Trichostrongylus spp. e Ostertagia spp. de origem ovina.

${ }^{1}$ Médico Veterinário, Pesquisador, Mestre, Empresa de Pesquisa Agropecuária e Extensão Rural de Santa Catarina S/A, Epagri Lages, CP 181, 88502-970, Lages, SC, E.mail: itaqui@epagri.rct-sc.br

${ }^{2}$ Médico Veterinário, Professor, PhD, Universidade do Estado de Santa Catarina - Centro de Ciências Agroveterinárias - Universidade do Estado de Santa Catarina. 
Na região Sul do Brasil, no Rio Grande do Sul, DOS SANTOS \& FRANCO (1967) e DOS SANTOS \& GONÇALVES (1967/1968) relataram a ocorrência de estirpes de $\boldsymbol{H}$. contortus resistentes ao thiabendazole. Posteriormente, SANTIAGO \& COSTA (1979) constataram resistência deste parasita ao levamisole. Estirpes de T. colubriformis resistente ao levamisole e ao tetramisole foram também relatadas (SANTIAGO et al., 1977 e 1978; SANTIAGO \& COSTA, 1979). Uma estirpe de $\boldsymbol{O}$. circumcincta, isolada no campo, resistente a doses de $7,5 \mathrm{mg} / \mathrm{kg}$ de levamisole também foi descrita por SANTIAGO et al. (1979).

$\mathrm{Na}$ década de 80 foi lançado no Brasil um terceiro grupo anti-helmíntico, as ivermectinas. Esta família apresentava como característica principal a alta eficácia contra a maioria dos helmintos gastrintestinais e até contra alguns parasitos externos, porém esta droga também não resistiu ao uso freqüente.

ECHEVARRIA \& TRINDADE (1989) verificaram que, em um rebanho ovino, medicado a cada vez que o exame de fezes apresentava em média 500 OPG, o $\boldsymbol{H}$. contortus tornou-se resistente ao ivermectin depois de 4,5 anos. Sobre a prevalência de resistência anti-helmíntica no Brasil, ECHEVARRIA \& PINHEIRO (1989) examinaram 31 rebanhos no município de Bagé-RS. Baseados em testes de redução de OPG e cultura de larvas conduzidos nos dias 0 e 7 pós-tratamento com thiabendazole ou tetramisole, esses autores encontraram $38,7 \%$ dos rebanhos com helmintos resistentes aos benzimidazóis, $25,8 \%$ ao tetramisole e $19,4 \%$ com resistência múltipla; somente $16,1 \%$ dos rebanhos examinados apresentaram helmintos sensíveis a ambas as drogas. Larvas que sobreviveram ao tratamento com thiabendazole eram principalmente Haemonchus spp enquanto aquelas sobreviventes ao tratamento com tetramisole eram Trichostrongylus spp. e Ostertagia spp.

No Paraná, SOUZA et al. (1997) verificaram em 25 rebanhos ovinos que a resistência anti-helmíntica estava presente em 92,3\% dos rebanhos testados para oxfendazol, em $80 \%$ para levamisole, em $85,7 \%$ para o tetramisol, em $91,3 \%$ para o ivermectin, em $30,8 \%$ para o moxidectin, em $85,8 \%$ para o closantel e em $87,5 \%$ para as associações tetramisol + disofenol e oxfendazol + closantel. CUNHA FILHO et al. (1999) avaliaram 10 rebanhos ovinos na região de Londrina, verificando que o fenômeno de resistência ocorreu em todas as propriedades com percentual de $100 \%$, $80 \%$ e $20 \%$ respectivamente para o albendazole, ivermectin e moxidectin. O gênero Haemonchus spp. foi o mais freqüente.
Em Santa Catarina, VICENTINI et al. (1993) testando a eficácia de alguns anti-helmínticos em quatro propriedades do município de Lages, por OPG mais percentagem larval, relataram que possivelmente ocorreu resistência de Haemonchus spp ao albendazole, oxfendazole e closantel em duas propriedades e ao ivermectin em uma e os gêneros Ostertagia spp e Trichostrongylus spp ao albendazole e oxibendazole em uma das propriedades. SOUZA et al. (1996) relataram a resistência do Haemonchus contortus ao ivermectin.

A comercialização de ovinos facilita a propagação da resistência de uma região para outra. Exemplo disto é o relato de VIEIRA et al. (1992), no Ceará, os quais observaram a presença de $\boldsymbol{H}$. contortus resistentes ao ivermectin e ao netobimin em ovinos provenientes do Paraná e Rio Grande do Sul.

Com objetivo de verificar a situação da resistência de helmintos gastrintestinais de ovinos a anti-helmínticos, no estado de Santa Catarina, realizou-se o presente trabalho.

\section{MATERIAL E MÉTODOS}

Foi avaliada a atuação dos antihelmínticos a base de ivermectin $(0,2 \mathrm{mg} / \mathrm{kg})$, levamisole $(10 \mathrm{mg} / \mathrm{kg})$, closantel $(10 \mathrm{mg} / \mathrm{kg})$ e albendazole $(10 \mathrm{mg} / \mathrm{kg})$, todos por via oral, em 65 rebanhos ovinos do estado de Santa Catarina. O trabalho foi realizado de outubro de 1997 a maio de 2000. A área de abrangência das avaliações foi em todas as regiões do estado de Santa Catarina, que têm produção ovina (Planalto Sul, Planalto Norte, Meio Oeste e Alto Vale do Itajaí). A escolha das propriedades foi feita por técnicos da Empresa de Pesquisa Agropecuária e Extensão Rural de Santa Catarina - S/A EPAGRI e da Companhia Integrada de Desenvolvimento Agrícola do Estado de Santa Catarina - CIDASC.

Foram realizadas duas visitas para cada propriedade. $\mathrm{Na}$ primeira, foram coletadas 10 amostras de fezes, avaliado o número de ovos por grama de fezes, OPG, (GORDON \& WHITLOCK, 1939) e preenchido um questionário, relacionado aos controles parasitários e à população ovina por propriedade. Quando a média atingiu acima de 500 OPG, este grupo era considerado controle e executava-se então o cultivo (ROBERTS \& O'SULLIVAN, 1950) e identificação de larvas. A seguir, foram aleatoriamente formados quatro grupos de 10 animais, identificados com tintas de diferentes cores, recebendo cada grupo, um tratamento anti-helmíntico conforme descrito acima. 
Na segunda visita, sete a 10 dias após os tratamentos, foram coletadas amostras de fezes dos animais de cada grupo, para a realização de OPG, cultivo e posterior identificações de larvas. Os resultados destes exames foram comparados com os do lote controle, para verificar a redução da média do OPG de nematóides gastrintestinais. Considerou-se como resistência, quando a eficácia da droga foi menor do que $95 \%$ e o intervalo de confiança inferior, menor que $90 \%$; baixa resistência, quando a eficácia foi superior a 95\% com intervalo de confiança menor que

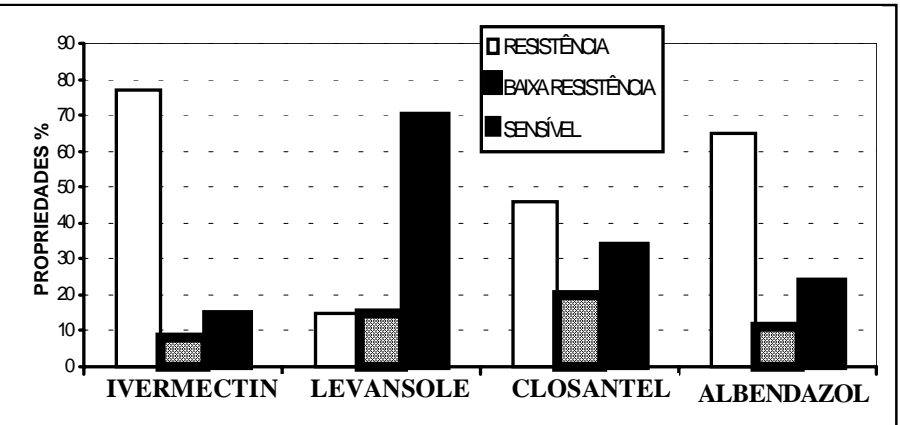

Figura 1 - Percentagem de propriedades em Santa Catarina, com helmintos de ovinos resistentes, com baixa resistência e sensíveis aos antihelmínticos, período de outubro/1997 a maio/2000.

dos rebanhos ao tratamento com tetramisole. A literatura mundial concorda que, para o levamisole, é mais comum observar a resistência de $\boldsymbol{T}$. colubriformis (MARTIN et al., 1990 e WALLER $\boldsymbol{e}$ t al., 1995).

Considerando que o closantel tem suas

De outubro de 1997 a maio de 2000, foram executadas as avaliações do teste de resistência de helmintos aos anti-helmínticos em 7.529 ovinos de 65 propriedades, representando $3 \%$ da população ovina, em 22 municípios localizados nas regiões do Planalto Serrano, Planalto Norte, Alto Vale do Itajaí e Centro Oeste do estado de Santa Catarina.

A resistência de Haemonchus spp. ao ivermectin $(0,2 \mathrm{mg} / \mathrm{kg})$, foi verificada em $77 \%$ das 65 propriedades (Figuras $1 \mathrm{e} 2$ ), principalmente naquelas que utilizavam de 8 a 12 medicações ao ano. ECHEVARRIA \& TRINDADE (1989) no Rio Grande do Sul, VIEIRA et al. (1992), no Ceará e SOUZA et al. (1996), em Santa Catarina, comprovaram resultados semelhantes. No Paraná, SOUZA et al. (1997) verificaram em 25 rebanhos resistência ao ivermectin, no entanto os autores não citaram os gêneros ou as espécies resistentes. CUNHA FILHO et al. (1997), neste mesmo Estado, observaram resultados semelhantes na região de Londrina onde o gênero mais prevalente foi Haemonchus spp.

Resistência de helmintos ao levamisole (10 $\mathrm{mg} / \mathrm{kg}$ ), foi observada em $15 \%$ das propriedades e baixa resistência em outras 15\% (Figura 1). Os gêneros mais prevalentes foram Trichostrongylus spp. (44\%) e Ostertagia spp. (39\%), concordando com os achados de SANTIAGO \& COSTA (1979) e SANTIAGO et al. (1979) para $\boldsymbol{T}$. colubriformis e $\boldsymbol{O}$. circumcincta respectivamente, no Rio Grande do Sul. Da mesma forma, ECHEVARRIA \& PINHEIRO (1989) verificaram também resistência de Trichostrongylus spp. e de Ostertagia spp. em 25,8\% limitações no controle de alguns helmintos, tais como Trichostrongylus spp. e Ostertagia spp., foram considerados como resistência somente os rebanhos em que houve predominância de Haemonchus spp., nos quais se verificou 13\% de casos (Figura 2). No Rio Grande do Sul, COSTA et al. (1997) e, em Santa Catarina, VICENTINI $\boldsymbol{e t}$ al. (1993) comprovaram a resistência do Haemonchus spp. ao closantel, da mesma forma que os achados do presente trabalho.

A resistência, principalmente de $\boldsymbol{H}$. contortus para o grupo dos benzimidazóis em ovinos, foi verificada na maioria dos países que se dedicam a ovinocultura. Exemplo destes são os trabalhos de LE JAMBRE (1978), LE JAMBRE $\boldsymbol{e} t$ al. (1979), na Austrália, de THEODORÍDES et al. (1970) e ANDERSEN \& CHRISTOFFERSON (1973), no Texas. Em Santa Catarina, foi detectada também resistência de Haemonchus spp. ao albendazole em $74 \%$ das propriedades avaliadas (Figuras 1 e 2), em acordo com os trabalhos de DOS SANTOS \& FRANCO, (1967) e DOS SANTOS \& GONÇALVES (1967/68) utilizando-se tiabendazole em ovinos, no Rio Grande do Sul. CUNHA FILHO et al. (1997) verificaram $100 \%$ de resistência ao albendazole em 10 rebanhos no Paraná, com predominância do gênero Haemonchus spp. Com relação a Ostertagia spp. e Trichostrongylus spp., $15 \%$ e $11 \%$ das propriedades respectivamente apresentavam ovinos com helmintos resistentes (Figura 1) para o albendazole. Dados semelhantes foram observados por HONG et al. (1996), na Inglaterra com relação a $\boldsymbol{O}$. circumcincta. 


\section{CONCLUSÕES}

Com base nestes resultados de avaliações de 65 rebanhos ovinos em Santa Catarina, pode-se concluir que a multirresistência estava presente na maioria dos rebanhos, dos quais $77 \%$ apresentavam-se com Haemonchus spp. resistentes ao ivermectin. Quanto ao albendazole, em $65 \%$ dos rebanhos verificou-se também a predominância de Haemonchus spp. resistente (74\%). O closantel encontrava-se em $13 \%$ das propriedades com resistência ao Haemonchus spp. Verificou-se 15\% de propriedades com resistência ao levamisol, predominando Trichostrongylus spp. (44\%) e Ostertagia spp. (39\%).

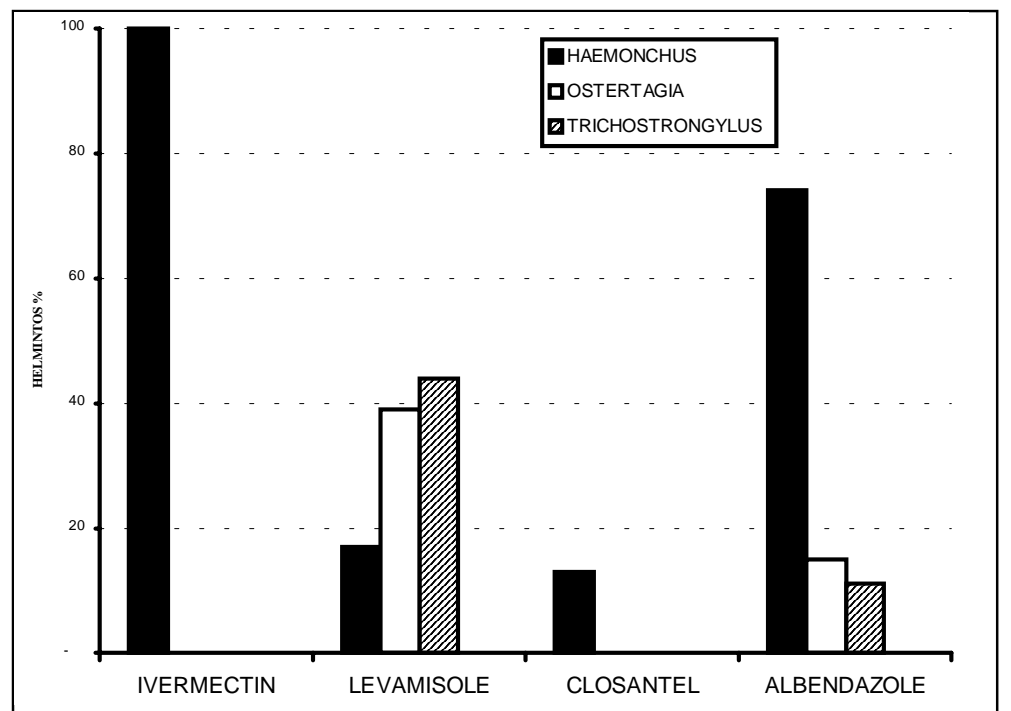

Figura 2 - Percentagem de helmintos resistentes aos diferentes princípios ativos em 65 propriedades de Santa Catarina, no período de outubro de 1997 a maio/2000.

\section{REFERÊNCIAS BIBLIOGRÁFICAS}

ANDERSEN, F.L., CHRISTOFFERSON, P.V. Efficacy of haloxon and thiabendazole against gastrointestinal nematodeos in sheep and goats en the Edwards Pleteau area of Texas. American Veterinary Research, v.34, n.11, p.1395-1398, 1973.

COSTA V.C. da, BENEVENGA, S.F., RUE M.L. de la. Avaliação, em ovinos da eficácia de uma mistura de albendazole e closantel, comparativamente a outros antihelmínticos contra Haemonchus contortus resistente. Revista Brasileira de Parasitologia Veterinária, v.6, n.2, suplemento 1, p.220, 1997.

CUNHA FILHO, L.F.C., YAMAMURA, M.H., PEREIRA, A.B.L. Resistência a anti-helmínticos em ovinos da região de Londrina. In: SEMINÁRIO BRASILEIRO DE PARASITOLOGIA VETERINÁRIA, 11. SEMINÁRIO DE PARASITOLOGIA VETERINÁRIA DOS PAÍSES DO MERCOSUL, 2. SIMPÓSIO DE CONTROLE INTEGRADO DE PARASITOS DE BOVINOS,1, 1999, Salvador, BA. Anais... Salvador : CBPV, 1999. p.153.

DOS SANTOS, V.T., FRANCO, E.G. O aparecimento de Haemochus resistente ao radical benzimidazole em Uruguaiana, RS. In: CONGRESSO LATINOAMERICANO DE PARASITOlOGiA, 1, 1967, Santiago, Chile. Anais... Santiago, Chile : Federation Latinoamericana de Parasitólogos, 1967. p.105.

DOS SANTOS, V.T., GONÇALVES, P.C. Verificação de estirpes de Haemonchus contortus resistentes ao thiabendazole no Rio Grande do Sul (Brasil). Revista da Faculdade Agronomia e Veterinária. v. 9, p.201-211, 1967/68.

ECHEVARRIA, F.A.M., PINHEIRO, A.C. Avaliação de resistência anti-helmíntica em rebanhos ovinos no município de Bagé, RS. Pesquisa Veterinária Brasileira, Rio de Janeiro, v.9, p.69-71, 1989.

ECHEVARRIA, F.A.M., TRINDADE, G.N.P. Anthelmintic resistance by Haemonchus contortus, to ivermectin in Brazil: A preliminary report. Veterinary Record, London, v.124, p.147-148, 1989.

GORDON, H. McL., WHITLOCK, H.V. A new technique for counting nematode eggs in sheep faeces. Journal Counc Scince Ind Australian, v.12, n.1, p.50-52, 1939.

HONG, C., HUNT, K.R., COLES, G.C. Ocurrence of anthelmintic resistant nematodes on sheep farms in England and goat farms in England an Wales. Veterinary Record, v.139 p. $83-86,1996$.

IBGE. Instituto Brasileiro de Geografia e Estatística, Censo Agropecuário, Santa Catarina, 1995-1996. Rio de Janeiro, 1997. N.21. 286p.

LEJAMBRE, L.F. Anthelmintics resistance in gastrointestinal nematodes of sheep. In: DONALD, A.D., SOUTHCOTT, W.H., DINNEEN, J.K. (Eds). The epidemiology and control of gastrointestinal parasites of sheep in Australia. Melbourne, Austrália : CSIRO, Division of Animal Health, 1978. p. $109-120$.

LEJAMBRE, L.F., MARTIN, P.J., WEBB, R.F. Thiabendazole resistance in field populations of Haemonchus contortus. Australian Veterinary Journal, v.55, n.4, p.163-166, 1979.

MARTIN, P.J., MACKENZIE, J.A., STONE, R.A. Levamisole resistence in Trichostrongylus colubriformis: A rexlinked recessive caracter. International Journal for Parasitology, v.20, p.867-872, 1990.

ROBERTS, F.H.S., O'SULLIVAN, P.J. Methods for eggs counts and larval cultures for strongyles infecting the gastrointestinal tract of catlle. Australian Journal Agriculture Research, v.1, n.1, p.99-102. 1950.

SANTIAGO M.A.M, COSTA, U.C, BENEVENGA, S.F. Trichostrongylus colubriformis resistentes ao levamisole. Revista do Centro de Ciências Rurais, Santa Maria, v.7, p.421-422, 1977.

SANTIAGO, M.A.M., COSTA, U.C., BENEVENGA, S.F. Atividade anti-helmíntica do dl-tetramisole e do thiabendazole em uma estirpe de Trichostrongylus colubriformis resistente ao levamisole. Revista do Centro de Ciências Rurais, Santa Maria, v.8, p.257-261, 1978. 
SANTIAGO, M.A.M., COSTA, U.C., BENEVENGA, S.F. Haemonchus contortus e Ostertagia circumcincta resistente ao levamisole. Revista do Centro de Ciências Rurais, Santa Maria, v.9, p.101-103, 1979.

SANTIAGO, M.A.M., COSTA, V.C. Resistência de Haemonchus contortus, Trichostrongylus colubriformis e Ostertagia spp., ao levamisole. Revista do Centro de Ciências Rurais, Santa Maria, v.9, p.315-318, 1979.

SOUZA, A.P. de., BELLATO, V., RAMOS, C.I. Resistência do endoparasita Haemonchus contortus ao ivermectin e ao albendazole em um rebanho ovino. Revista Agropecuária Catarinense, Florianópolis, v.9, n.1, p.38-39, 1996.

SOUZA, F.P. de, THOMAS-SOCCOL, V., CASTRO, $\boldsymbol{e} \boldsymbol{t} \boldsymbol{a l}$ Contribuição para o estudo da resistência de helmintos gastrointestinais de ovinos (Ovis aries) aos anti-helmínticos, no Estado do Paraná. Revista Brasileira de Parasitologia Veterinária, v.6, n.2. suplemento 1, p.217, 1997.

THEODORIDES, V.J., SCOTT, G.C., LANDERMAN, M. Strains of Haemonchus contortus resistant against benzimidazole anthelmintic American Journal of Veterinary Research, v.31, n.5, p.857-863, 1970

WALLER, P.J., DASH, K.M., BARGER, I.A. et al. Anthelmintic resistance in nematode parasites of sheep: learning from the Australian experience. Veterinary Record, v.136, p.411-413, 1995.

VIEIRA, L.S., BERNE. M.E.A., CAVALCANTE, $\boldsymbol{e} \boldsymbol{t} \boldsymbol{a l}$ Haemonchus contortus resistance to ivermectin and netobimin in Brazilian sheep. Veterinary Parasitology. v.45, p.111-116, 1992.

VICENTINI, A., SOUZA, A.P. de, BELLATO, V. Avaliação de eficácia de alguns anti-helmínticos utilizados para o controle de nematodeos gastrintestinais de ovinos. In: SEMINÁRIO CATARINENSE DE INICIAÇÃO CIENTÍFICA, 3. 1993, Florianópolis, SC. Anais... Florianópolis : UDESC, 1993. p.157.

VIZARD, A.L., WALLACE, R.J. A simplified faecal egg count reduction test. Australian Veterinary Journal v.64, n.4, p.109-111, 1987. 